\title{
ON THE QUALITATIVE BEHAVIOUR OF PARAMETER DEPENDENT SYSTEMS
}

\author{
SÁNDOR KOVÁCS AND SZILVIA GYÖRGY
}

Received 05 November, 2018

\begin{abstract}
In the first part of this work we present some basics about the connection of exponential dichotomy and Floquet-theory. Then we study some stability notions of linear differential equations based on the concept of the exponential and ordinary dichotomy. More precisely we shall show that the definition of the asymptotical (Lyapunov-) stability can be formulated by using the exponential dichotomy. In the main part of the paper we consider parameter dependent non-autonomous linear and autonomous nonlinear systems which have at a certain value of the parameter an asymptotically stable solution. Then we examine the question how much the parameter can be changed such that the solution remains asymptotically stable. Finally, we study the orbital stability of periodic solutions of autonomous systems applying the previous results.
\end{abstract}

2010 Mathematics Subject Classification: 34D09; 34D20

Keywords: Exponential dichotomy, Lyapunov-stability, orbital stability, Floquet theory

\section{INTRODUCTION}

In many fields of the applied mathematics it may often happen that when we would like to study a phenomenon when we don't know the exact mathematical model or the system of the differential equations which describe the given phenomenon. For example it may happen that there are some parameters in the mathematical model, which depend on the variables of the system or on environmental factors etc. In many cases the exact values of these parameters are not known because we can just measure or estimate them, or even although if we can calculate somehow the exact values of the parameters but we can not give their exact value numerically. In these cases we can study the qualitative behaviour of the model with the estimated values of the parameters. Therefore, it is a legitimate expectation that the qualitative behaviour of the model with the estimated parameters must be the same as the behaviour of the exact model. However, often we couldn't verify the correspondence of the qualitative properties of these two models. But we can get bounds and intervals for the parameters such that we can prove theoretically that if the parameters are within these

The authors were supported in part by the European Union, co-financed by the European Social Fund (EFOP-3.6.3-VEKOP-16-2017-00002). 
bounds or intervals, then the behaviour of the unknown functions in the models do not change, which means that the estimated model reflects faithfully the exact model, and consequently, the studied phenomenon, too.

The objective of this paper is to study the Lyapunov stability of the solutions of system of differential equations in the form

$$
\dot{x}=f(i d, x, \mu),
$$

where $\mu$ is a parameter. More precisely, we would like to know that if with a certain value of the parameter $\mu_{0}$ a solution of system (1.1) is asymptotically stable, then under what conditions we can prove that the solution remains asymptotically stable, if the parameter changes are sufficiently small.

In this paper we are going to use the notion of the exponential dichotomy and two results of the theory of the exponential dichotomy: the relation between the exponential dichotomy and the stability of linear systems, and a theorem about the roughness of the exponential dichotomy.

It is well known that the qualitative properties of the solutions of systems of ordinary differential equations depend continuously on the parameters of the right hand side. In this paper we use the robustness of the exponential dichotomy in order to show that the stability properties of the system change continuously.

The paper is organized as follows: in Section 2 we present the basic notions and theorems with respect to the exponential dichotomy and the Floquet theory, which we will use in the next section, then in Section 3 we are going to study the stability of different types of solutions of system (1.1): firstly the stability of (the trivial solution) of linear systems, then the stability of the equilibrium point of autonomous (but not necessarily linear) systems, and finally the stability of periodic solutions of autonomous systems.

\section{MATHEMATICAL BACKGROUND: THE USED FRAMEWORK AND TOOLS}

\subsection{Exponential dichotomy}

In the theory of the exponential dichotomy we study systems of linear differential equations. There are a lot of papers where the authors study linear systems in general cases, for example with arbitrary finite dimensional phase space or infinite phase space (cf. [5]). In this paper we use simply the usual phase space $\left(\mathbb{R}^{n},\|\cdot\|\right)$, where $\|\cdot\|$ denotes an arbitrary vector norm. Let us consider the linear system

$$
\dot{x}=A x,
$$

where $A \in \mathfrak{C}\left(J, \mathbb{R}^{n \times n}\right)$ with a fixed dimension $n \in \mathbb{N}$ and interval $J:=[\tau,+\infty)$, where $\tau \in \mathbb{R}$. Let us denote the fundamental matrix of system (2.1) by $\Phi$, i.e. let $\Phi$ be a regular matrix solution of (2.1). Thus, the entire solution $\varphi$ of system (2.1) with an initial condition $x(\tau)=\xi$ has the form

$$
\varphi(t)=\Lambda(t, \tau) \xi:=\Phi(t) \Phi^{-1}(\tau) \xi \quad(t \in J)
$$


where $\Lambda(\cdot, \tau)$ denotes the Cauchy matrix of (2.1).

In this paper we will use the definition of the exponential dichotomy in the sense discussed in the book of Coppel (cf. [1]).

Definition 1 (cf. [1]). The equation (2.1) is said to possess an exponential dichotomy if there exist a projection $P$ and positive constants $K_{1}, K_{2}, \alpha_{1}, \alpha_{2}$ such that

$$
\begin{aligned}
&\left\|\Phi(t) P \Phi^{-1}(s)\right\| \leq K_{1} e^{-\alpha_{1}(t-s)} \quad(t \geq s), \\
&\left\|\Phi(t)(I-P) \Phi^{-1}(s)\right\| \leq K_{2} e^{-\alpha_{2}(s-t)} \quad(s \geq t) .
\end{aligned}
$$

It is said to possess an ordinary dichotomy if the inequalities (2.2) hold with $\alpha_{1}=$ $\alpha_{2}=0$.

In the autonomous case, i. e. when $A$ is a constant matrix, we can easily determine the existence of the exponential dichotomy by looking for the eigenvalues of the coefficient matrix.

Proposition 1 (cf. [1], [6]). Let $A \in \mathbb{R}^{n \times n}$ be a matrix and let us consider the autonomous system

$$
\dot{x}=A x .
$$

It has an exponential dichotomy on $J:=[0,+\infty)$ if and only if no eigenvalue of the constant matrix $A$ has zero real part. It has an ordinary dichotomy, if and only if all eigenvalues of $A$ with zero real part are semisimple (which means that these eigenvalues are simple roots of the minimal polynomial of $A$ ). In each case we can take the projection $P$ as the spectral projection defined by

$$
P:=\frac{1}{2 \pi \imath} \int_{\gamma}(z I-A) \mathrm{d} z .
$$

To prove the main results of this paper we are going to use the following theorems: the first states the roughness of the exponential dichotomy, the second gives the relation between the exponential dichotomy and the Lyapunov-stability of linear systems.

Theorem 1 (cf. [4]). Let us assume that the linear system with continuous coefficients

$$
\dot{x}=A x
$$

possesses an exponential dichotomy on $J:=[0,+\infty)$ with some constants $K_{1}, K_{2}$, $\alpha_{1}, \alpha_{2}>0$ and projection $P$. Let $B$ a matrix valued function with continuous coefficients defined on the interval $J$. If for the function $B$ the inequality

$$
\limsup _{s \rightarrow+\infty}\|B(s)\|<\left(\frac{K_{1}}{\alpha_{1}}+\frac{K_{2}}{\alpha_{2}}\right)^{-1}
$$


holds, then system

$$
\dot{x}=(A+B) x .
$$

possesses an exponential dichotomy on the interval $J$ with projection $Q$, where $Q$ is similar to $P$.

In Definition 1 we can assume that $K_{1}=K_{2}=: K$ and $\alpha_{1}=\alpha_{2}=: \alpha$, and if we use these constants, the upper bound at condition (2.6) is the following:

$$
\limsup _{s \rightarrow+\infty}\|B(s)\|<\frac{\alpha}{2 K} \text {. }
$$

In Section 3 the other key theorem will be the following result, which connects the notion of the exponential dichotomy and the stability of linear systems.

Theorem 2 (cf. [2]). The linear system (2.1) is

(1) asymptotically stable if and only if it admits an exponential dichotomy with projection $P=I$, furthermore

(2) stable if and only if it admits an ordinary dichotomy with projection $P=I$.

\subsection{Some basics from the Floquet-theory}

In the last part of Section 2 we are going to study the stability of periodic orbits (of parameter dependent systems) using some tools from the Floquet theory, therefore in this subsection we summarize the necessary results about the stability of periodic orbits and some relevant fields.

Let $A \in \mathfrak{C}\left(\mathbb{R}, \mathbb{R}^{n \times n}\right)$ be a matrix-valued function, for which $A(t+T)=A(t)$ is fulfilled for each $t \in \mathbb{R}$ with some period $T>0$. Let us consider the linear system with periodic coefficient matrix

$$
\dot{x}=A x .
$$

Let us denote by $C$ the principal or monodromy matrix of (2.9):

$$
C:=\Lambda(T, 0) \text {. }
$$

Following from the well-known Floquet Theorem, we can write the Cauchy matrix of system (2.9) in a special form.

Theorem 3 (Floquet). The fundamental matrix of (2.9) can be written in the form

$$
\Lambda(t, 0)=P(t) e^{B t} \quad(t \geq 0),
$$

where $P \in \mathfrak{C}^{1}\left([0,+\infty), \mathbb{R}^{n \times n}\right)$ is a regular $T$-periodic matrix, $P(0)=I$ and $B \in$ $\mathbb{R}^{n \times n}$ is a constant matrix.

The eigenvalues of these two matrices $C$ and $B$ play an important role at the investigation of the stability of system (2.9). The eigenvalues of the matrix $C$ are the characteristic multipliers of the system (2.9), and the eigenvalues of the matrix $B$ are the characteristic exponents of the system (2.9). Following from the identity

$$
e^{B T}=I \cdot e^{B T}=P(T) \cdot e^{B T}=\Lambda(T, 0)=C,
$$


if $v$ is a characteristic exponent, then $\lambda=e^{\nu T}$ is a characteristic multiplier and vice versa. Hence, by $T>0$

$$
|\lambda|<1 \quad \Longleftrightarrow \quad \Re(v)<0 .
$$

Let us assume that 1 is a simple characteristic multiplier of system (2.9) and there is no other characteristic multiplier on the unit circle. Then, the fundamental matrix of the system has the following form

$$
\Phi(t)=P(t)\left[\begin{array}{cc}
1 & 0 \\
0 & \exp \left(B_{1} t\right)
\end{array}\right] \quad(t \in \mathbb{R}),
$$

where $P$ is regular, continuously differentiable, periodic matrix valued function with period $T$, and $B_{1} \in \mathbb{R}^{(n-1) \times(n-1)}$ is a hyperbolic matrix.

Let $\Omega \subset \mathbb{R}^{n}$ be an open, connected set, $f \in \mathfrak{C}^{1}(\Omega)$ and let us consider the system

$$
\dot{x}=f(x) \text {. }
$$

Let us assume that $\varphi: \mathbb{R} \rightarrow \mathbb{R}^{n}$ is a nontrivial, $T$-periodic solution of the system. We know that the variational system

$$
\dot{x}=(\partial f \circ \varphi) x
$$

has a $T$-periodic solution if and only if 1 is a characteristic multiplier of the system. The theorem of Andronov and Witt gives a sufficient condition for the asymptotical stability of the periodic solution $\varphi$.

Theorem 4 (Andronov-Witt, see [3]). If 1 is a simple characteristic multiplier of the system (2.12) and the remaining $n-1$ characteristic multipliers are in modulus less then 1, then the periodic solution $\varphi$ is orbitally asymptotically stable.

The Andronov-Witt Theorem has a version which states the instability of the periodic solutions.

Theorem 5 (cf. [3]). If at least one of the characteristic multipliers of the variational system is in modulus greater than 1, then the periodic solution $\varphi$ is orbitally unstable.

Let us assume that $\varphi$ is orbitally asymptotically stable, 1 is a simple characteristic multiplier and there is no other characteristic multiplier on the complex unit circle, i.e. if $M \subset \mathbb{C}$ denotes the set of the characteristic multipliers, then

$$
M=\{1\} \cup\{\lambda \in \mathbb{C}: \lambda \text { is a characteristic multiplier, }|\lambda| \neq 1\} .
$$

If there would be a characteristic multiplier $\lambda \in M$ such that $|\lambda|>1$, by Theorem 4 it would contradict the fact that $\varphi$ is asymptotically stable. Hence, because we assumed that there are no characteristic multiplier on the unit circle except 1, we proved that the remaining $n-1$ characteristic multipliers are in modulus less than 1 . This means that in this case the conversion of the Andronov-Witt Theorem 4 is also true. 
Let us see how the variational system looks like, in the case when the periodic solution $\varphi$ is orbitally asymptotically stable, and hence 1 is a characteristic multiplier, and there are no other characteristic multipliers on the complex unit circle. Then, by Floquet Theorem 3, with a suitable invertible matrix $S \in \mathbb{R}^{n \times n}$ we have

$$
S^{-1} C S=\left[\begin{array}{cc}
1 & 0 \\
0 & C_{1}
\end{array}\right],
$$

where $C_{1} \in \mathbb{R}^{(n-1) \times(n-1)}$ is such that $\exp \left(B_{1} T\right)=C_{1}$ is fulfilled with some matrix $B_{1}$. Let us introduce one more notation, namely

$$
B:=\left[\begin{array}{cc}
0 & 0 \\
0 & B_{1}
\end{array}\right] \text {. }
$$

With the above matrix $B$ the equality

$$
\exp (B t)=\left[\begin{array}{cc}
1 & 0 \\
0 & \exp \left(B_{1} t\right)
\end{array}\right]
$$

is satisfied, and hence

$$
S^{-1} C S=e^{B T}
$$

is also true.

Let us study a little bit more the second assumption of the Andronov-Witt Theorem (the remaining characteristic multipliers are in modulus less then 1). By (2.10) this assumption means that the eigenvalues of the matrix $B_{1}$ have negative real part, or in other words, the $n-1$ dimensional autonomous linear system

$$
\dot{y}=B_{1} y
$$

is asymptotically stable, i.e. this system possesses an exponential dichotomy with projection $P=I \in \mathbb{R}^{(n-1) \times(n-1)}$.

In conclusion, if $\varphi$ is a periodic solution of system (2.11), 1 is a simple characteristic multiplier and the other characteristic multipliers are in modulus less or greater than 1 , then $\varphi$ is asymptotically stable if and only if system (2.14) possesses an exponential dichotomy with projection $P=I$.

\section{MAIN RESULTS}

\subsection{Nonautonomous linear systems}

In the first application we are going to consider the stability of the system

$$
\dot{x}=A(\cdot ; \mu) x,
$$

where $A \in \mathfrak{C}^{1}\left(\mathbb{R} \times \mathbb{R}^{p}, \mathbb{R}^{n \times n}\right), \mu \in \mathbb{R}^{p}$ represents the parameter of the system, $p \in$ $\mathbb{N}$. We are going to investigate the following problem: if for a certain value of the parameter $\mu_{0}$ system (3.1) is asymptotically stable, then how much we can change the value of the parameter such that the stability of the system does not change. For this we are going to use Theorem 1 on exponential dichotomy. 
Theorem 6. Let us assume that the coefficient matrix A of (3.1) is Lipschitz continuous w.r.t. the second variable on the positive half line, i.e. there exists a constant $L>0$ such that for each $\mu_{1}$ and $\mu_{2}$

$$
\left\|A\left(t, \mu_{1}\right)-A\left(t, \mu_{2}\right)\right\| \leq L\left\|\mu_{1}-\mu_{2}\right\|
$$

is satisfied with arbitrary $t \geq 0$. We assume that for $\mu_{0} \in \mathbb{R}^{p}$ system (3.1) is asymptotically stable or equivalently, it has an exponential dichotomy on $J=[0,+\infty)$ with constants $K, \alpha>0$ and projection $P=I$. Then for every parameter $\lambda \in \mathbb{R}^{p}$ for which

$$
\left\|\mu_{0}-\lambda\right\| \leq \frac{1}{L} \cdot \frac{\alpha}{2 K}
$$

holds, system (3.1) is asymptotically stable with $\lambda$.

Proof. Let us assume that with the parameter value $\mu_{0}$ the system

$$
\dot{x}=A_{0} x
$$

is asymptotically stable, where $A_{0}:=A\left(\cdot, \mu_{0}\right)$. Then we know from Theorem 2 that this system possesses an exponential dichotomy with projection $P=I$ and with constants $K, \alpha>0$. Let $\lambda$ be a parameter value such that the condition (3.2) of Theorem 6 is fulfilled, i. e.

$$
\left\|\mu_{0}-\lambda\right\| \leq \frac{1}{L} \cdot \frac{\alpha}{2 K}
$$

holds. Then let us write the matrix $A(t, \lambda)$ in the following form:

$$
A(t, \lambda)=A\left(t, \mu_{0}\right)+\left(A(t, \lambda)-A\left(t, \mu_{0}\right)\right),
$$

and define the function $B$ as

$$
B(t):=A(t, \lambda)-A\left(t, \mu_{0}\right) \quad(t \geq 0) .
$$

Hence, by using the perturbation Theorem 1, the system

$$
\dot{x}(t)=\left(A\left(t, \mu_{0}\right)+B(t)\right) x(t) \quad(t \geq 0)
$$

has an exponential dichotomy, too, with projection $Q$, because for each $t \geq 0$ we know from the assumptions that

$$
\|B(t)\|=\left\|A(t, \lambda)-A\left(t, \mu_{0}\right)\right\| \leq L\left\|\lambda-\mu_{0}\right\| \leq \frac{\alpha}{2 K} .
$$

So we proved that the system

$$
\dot{x}(t)=\left(A\left(t, \mu_{0}\right)+A(t, \lambda)-A\left(t, \mu_{0}\right)\right) x(t)=A(t, \lambda) x(t) \quad(t \geq 0)
$$

admits an exponential dichotomy with projection $Q$. By the perturbation Theorem 1 we know that the two projections $P=I$ and $Q$ are similar, which means that $Q=c \cdot I$, where $c \in \mathbb{R}$ is a constant. Then we get that the perturbed system (3.3) has an exponential dichotomy, too, with projection $\tilde{Q}=I$, and as a consequence we get that system (3.3) is asymptotically stable. 
Assume now that system (3.1) is autonomous, i.e. for the coefficient function $A \in \mathfrak{C}^{1}\left(\mathbb{R}^{p}, \mathbb{R}^{n \times n}\right)$ holds and with a certain value $\mu_{0}$ of the parameter the autonomous system

$$
\dot{x}=A\left(\mu_{0}\right) x
$$

is asymptotically stable. Then we know that all eigenvalues of the coefficient matrix $A\left(\mu_{0}\right) \in \mathbb{R}^{n \times n}$ have negative real part or equivalently (cf. Proposition 1 or Theorem 2) the system possesses an exponential dichotomy with projection $P=I$ (and with constants $K, \alpha>0$ ). Applying Theorem 6, we obtain that system

$$
\dot{x}=A(\lambda) x
$$

is asymptotically stable, too, if condition (3.2) is fulfilled for $\lambda$. It follows that with these $\lambda$ values of the parameters all eigenvalues of matrix $A(\lambda)$ have negative real parts. It means that this way we get a condition under which the eigenvalues of a matrix (which depends on a parameter) remain in the left half plane. (It is easy to show that the real part of the eigenvalues of $A(\lambda)$ remain negative with small changes of the parameter $\lambda$ ).

3.2. The stability of an equilibrium point of a system of nonlinear autonomous equations

Let us consider the autonomous system

$$
\dot{x}=f(x ; \mu)
$$

where $f \in \mathfrak{C}^{1}\left(\mathbb{R}^{n} \times \mathbb{R}^{p}, \mathbb{R}^{n}\right)$ and $\mu \in \mathbb{R}^{p}$ is a parameter. Let us denote by $x_{0}(\mu)$ the equilibrium point of the system. Similarly to the previous subsection, we are going to study the following problem: if the equilibrium point is asymptotically stable then how much we can change the value of the parameter such that the equilibrium point remains asymptotically stable.

Theorem 7. Let us assume that $x_{0}(\mu)$ is an equilibrium of system (3.4) for each $\mu \in \mathbb{R}^{p}$, and there exists a $\mu_{0} \in \mathbb{R}^{p}$ such that $x_{0}\left(\mu_{0}\right)$ is an asymptotically stable equilibrium point. If the function $F$

$$
F(\mu):=\partial_{1} f\left(x_{0}(\mu) ; \mu\right) \quad\left(\mu \in \mathbb{R}^{p}\right)
$$

is Lipschitz continuous, i.e. there exists a constant $L>0$ such that for all parameters $\mu_{1}, \mu_{2} \in \mathbb{R}^{p}$

$$
\left\|F\left(\mu_{1}\right)-F\left(\mu_{2}\right)\right\| \leq L\left\|\mu_{1}-\mu_{2}\right\|
$$

is satisfied, then there is a constant $R>0$ such that for every

$$
\lambda \in B_{R}\left(\mu_{0}\right):=\left\{\mu \in \mathbb{R}^{p}:\left\|\mu-\mu_{0}\right\|<R\right\},
$$

$x_{0}(\lambda) \in \mathbb{R}^{n}$ is an asymptotically stable equilibrium point of (3.4). 
Proof. Let us assume that with the parameter value $\mu_{0}$ the equilibrium point $x_{0}\left(\mu_{0}\right)$ is asymptotically stable. This is equivalent to the fact that the linear autonomous system

$$
\dot{y}=\partial_{1} f\left(x_{0}\left(\mu_{0}\right) ; \mu_{0}\right) y
$$

is asymptotically stable, where $\partial_{1} f(\cdot, \mu)$ denotes the Jacobian matrix of the function $f$ w.r.t. the first variable. By Theorem 2 this is equivalent to the fact that the linear system possesses an exponential dichotomy with projection $P=I$. Hence we can apply the previous theorem, which says that there exists a constant $R \in \mathbb{R}$ such that if $\left\|\lambda-\mu_{0}\right\|<R$, then the system

$$
\dot{y}=\partial_{1} f\left(x_{0}(\lambda) ; \lambda\right) y
$$

is asymptotically stable, which means that the equilibrium point $x_{0}(\lambda)$ is also asymptotically stable.

Now we show an example: how the previous theorem works at a concrete system of differential equations.

Example 1. Let us consider the following system of differential equations of LotkaVolterra type:

$$
\left\{\begin{array}{l}
\dot{x}=x(a-x-y) \\
\dot{y}=y(b-2 x-y)
\end{array}\right.
$$

where $(a, b) \in \mathbb{R}^{2}$ is a parameter. (Assume that we have two populations, $x(t)$ and $y(t)$ denote the number of the individuals of the populations, further $a$ and $b$ are unknown rates, which depend on a lot of things, for example on environmental factors.) Let us study the stability of the positive equilibrium point

$$
\left(x_{0}, y_{0}\right):=(b-a, 2 a-b) .
$$

Let us denote by $f$ the function of the right hand side of the system:

$$
f(x, y):=\left[\begin{array}{c}
x(a-x-y) \\
y(b-2 x-y)
\end{array}\right] \quad(x, y \in \mathbb{R}) .
$$

The Jacobian of $f$ at $\left(x_{0}, y_{0}\right)$ is

$$
\partial f\left(x_{0}, y_{0}\right)=\left[\begin{array}{cc}
a-b & a-b \\
2 b-4 a & b-2 a
\end{array}\right]
$$

hence the function $F$ in Theorem 7 has the following form:

$$
F(a, b):=\left[\begin{array}{cc}
a-b & a-b \\
2 b-4 a & b-2 a
\end{array}\right] \quad(a, b \in \mathbb{R}) .
$$

We can easily prove that this function is Lipschitz continuous: for each $(a, b),\left(a_{1}, b_{1}\right) \in \mathbb{R}^{2}$ we have

$$
\left\|F(a, b)-F\left(a_{1}, b_{1}\right)\right\|_{1}=\left\|\left[\begin{array}{cc}
a-a_{1}-\left(b-b_{1}\right) & a-a_{1}-\left(b-b_{1}\right) \\
2\left(b-b_{1}\right)-4\left(a-a_{1}\right) & b-b_{1}-2\left(a-a_{1}\right)
\end{array}\right]\right\|_{1}
$$




$$
\begin{aligned}
& =2\left|b-b_{1}-2\left(a-a_{1}\right)\right|+\left|b-b_{1}-\left(a-a_{1}\right)\right| \\
& \leq 2\left(\left|b-b_{1}\right|+2\left|a-a_{1}\right|\right)|+| b-b_{1}|+| a-a_{1} \mid \\
& =3\left|b-b_{1}\right|+5\left|a-a_{1}\right| \\
& \leq 5\left\|(a, b)-\left(a_{1}, b_{1}\right)\right\|_{1} .
\end{aligned}
$$

Let us assume that $a=1 / 2$ and $b=1 / 3$ (for example let us imagine that we have two concrete populations and we get these estimations for the parameters from an experiment.) In this case

$$
\partial f\left(x_{0}, y_{0}\right)=\left[\begin{array}{cc}
\frac{1}{6} & \frac{1}{6} \\
-\frac{4}{3} & -\frac{2}{3}
\end{array}\right]
$$

and the eigenvalues are

$$
\lambda_{1,2}=-\frac{1}{4} \pm \frac{\sqrt{7}}{12} l
$$

which means that the equilibrium point is asymptotically stable. By Theorem 2 the linear system

$$
\dot{y}=\partial f\left(x_{0}, y_{0}\right) y
$$

possesses an exponential dichotomy with $P=I$. It is easy to calculate that the constants of the definition of the exponential dichotomy are $K=2$ and $\alpha=1 / 4$ at this system. Hence the previous Theorem 7 says that the equilibrium point is asymptotically stable with some other parameters $(\tilde{a}, \tilde{b}) \in \mathbb{R}^{2}$, if for the parameters $(\tilde{a}, \tilde{b})$

$$
\|(1 / 2,1 / 3)-(\tilde{a}, \tilde{b})\|_{1} \leq \frac{1}{L} \cdot\left(\frac{K_{1}}{\alpha_{1}}+\frac{K_{2}}{\alpha_{2}}\right)^{-1}=0.0125 .
$$

\subsection{The stability of periodic solutions}

In the last part of this section let us consider the system (3.4) again:

$$
\dot{x}=f(x, \mu)
$$

where $\mu \in \mathbb{R}^{p}$ and assume that $\varphi_{\mu}$ is a periodic solution of (3.7). Then we know that 1 is a characteristic multiplier of the variational system (2.12), furthermore we assume that there are no other characteristic multipliers on the unit circle. We have seen in Section 2.2 that in this case $\varphi_{\mu}$ is asymptotically stable if and only if the linear system

$$
\dot{y}=B_{1_{\mu}} y
$$

possesses an exponential dichotomy with projection $P=I$, where the matrix $B_{1_{\mu}} \in$ $\mathbb{R}^{(n-1) \times(n-1)}$ comes from (2.13) and is related to the monodromy matrix of the variational system of $\varphi_{\mu}$. With this observation we can apply the perturbation Theorem 1 referring to the stability of the exponential dichotomy, to study the stability of periodic orbits, similarly to the previous two subsections. 
Theorem 8. Let us denote by $\varphi_{\mu}$ a periodic solution of system (3.4) (with parameter value $\mu$ in a neighbourhood of some $\left.\mu_{0}\right)$. Let us assume that with a certain value of the parameter $\mu_{0}, \varphi_{\mu_{0}}$ is orbitally asymptotically stable, and $\lambda=1$ is a simple characteristic multiplier, and except for this, there is no other characteristic multiplier on the unit circle, consequently, the system

$$
\dot{y}=B_{1_{\mu_{0}}} y
$$

possesses an exponential dichotomy with some constants $K_{1}, K_{2}, \alpha_{1}, \alpha_{2}$ and projection $P=I$. Then, for each parameter value $\lambda \in \mathbb{R}^{p}$, for which

$$
\left\|B_{1_{\mu_{0}}}-B_{1_{\lambda}}\right\|<\left(\frac{K_{1}}{\alpha_{1}}+\frac{K_{2}}{\alpha_{2}}\right)^{-1}
$$

holds, the periodic solution $\varphi_{\lambda}$ is orbitally asymptotically stable.

Proof. Since $\varphi_{\lambda}$ is a periodic solution (if $\lambda$ is in a neighbourhood of $\mu_{0}$ ), we know that 1 is a characteristic multiplier, furthermore we assumed that the other characteristic multipliers are in modulus strictly less or greater than 1. Hence, if condition (3.8) holds, then the following system

$$
\dot{y}=B_{1_{\lambda}} y
$$

has an exponential dichotomy with projection $P=I$, which means that the matrix $B_{1_{\lambda}}$ has eigenvalues with negative real parts. Thus we know that with the parameter value $\lambda$ the other characteristic multipliers are in modulus less then 1 . Hence, by Theorem 4 the periodic solution $\varphi_{\lambda}$ is orbitally asymptotically stable.

\section{REFERENCES}

[1] W. A. Coppel, Dichotomies in stability theory. Berlin Heidelberg New York: Springer Verlag, 1978.

[2] S. Császár and S. Kovács, "Exponential dichotomy and the stability of linear systems." Annales Univ. Sci. Budapest., Sec. Comp., vol. 47, pp. 211-226, 2018.

[3] M. Farkas, Periodic Motions. New York: Springer-Verlag, 1994.

[4] N. Ju and S. Wiggins, "On Roughness of Exponential Dichotomy." J. Appl. Math. Anal. Appl., vol. 262, pp. 39-49, 2001, doi: doi:10.1006/jmaa.2001.7496.

[5] N. Lupa and M. Megan, "Exponential dichotomies of evolution operators in Banach spaces." Monats. Math., vol. 174, pp. 265-284, 2014, doi: DOI 10.1007/s00605-013-0517-y.

[6] C. Pötzsche, "Bifurcations in nonautonomous dynamical systems: Results and tools in discrete time." Proceedings of the International Workshop Future Directions in Difference Equations, pp. 163-212, 2011, June 13-17, Vigo, Spain, doi: 978-84-8158-541-4/pbk.

Authors' addresses

Sándor Kovács

Eötvös L. University, Department of Numerical Analysis, Pázmány Péter sétány 1/C, H-1117 Budapest, Hungary

E-mail address: alex@ludens.elte.hu 
Szilvia György

Eötvös L. University, Pázmány Péter sétány 1/C, H-1117 Budapest, Hungary

E-mail address: csaszarecs.elte.hu 